\title{
An experimental study on the enzymatic hydrolysis of cellulose*
}

\author{
Zhao-Chun $\mathrm{Wu}^{\dagger}$ \\ School of Urban Construction and Safety Engineering, \\ Shanghai Institute of Technology, \\ Shanghai, 201418, China \\ E-mail: wzcyy@sit.edu.cn
}

\begin{abstract}
The utilization of biomass energy has received increasingly attention. Converting industrial and agricultural production wastes into biomass energy to provide clean and sustainable energy has become a larger trend in energy utilization. Biological chemical conversion method, as one of the methods to obtain biomass energy, consists of deriving the sugary by biological decomposition and generating ethanol as energy products via the fermentation process. The decomposition rate and the glucose yield have significant influence on the process of biomass energy production. To reveal the effect on the hydrolysis process with different lignin, pretreatment methods of the samples, enzyme and hydrolysate concentration, the experimental research of the enzymatic hydrolysis of cellulose were conducted, and some characteristics are demonstrated and analyzed.

Keywords: Biomass Energy; Lignin; Enzymatic Hydrolysis.
\end{abstract}

\section{Introduction}

With rapid economic development, the demand of energy is constantly rising in recent decades. The use of fossil fuels which produce large amounts of $\mathrm{CO}_{2}, \mathrm{SO}_{2}$, $\mathrm{NO}_{\mathrm{x}}$, harmful gases, greenhouse gases and other pollutants, is causing great harm to the environment. Moreover, because of non-renewable and finite reserved fossil fuels, growing energy demand also poses a serious energy shortage. To ensure sustainable development of the modern society, adjusting energy structure and developing clean and renewable energy is an urgent task. Thus, biomass energy, as an alternative oil material, is booming. The theoretical and experimental researches have grown up to be a hot topic and received widely attention by scientists [1-5].

Biomass energy can be obtained by several methods, such as biological chemical conversion method. In the method, the lignocelluloses which come from waste materials is converted into sugars by the enzymatic hydrolysis firstly and then fermented to produce ethanol fuel. Thus hydrolytic process of biomass

\footnotetext{
" This work is supported by NSFC (No. 51576133).
} 
has significant influence on the biomass energy production. To reveal the effect on the hydrolysis process with different lignin, pretreatment methods of the samples, enzyme and hydrolysate concentration, the experimental research of the enzymatic hydrolysis of cellulose were conducted, and some characteristics are demonstrated and analyzed.

\section{The Experimental Materials}

Sample materials consist of straw, spruce and aspen. Owing to the cell walls of lignocelluloses biomass have natural resistance, called recalcitrance, to enzymatic deconstruction, therefore, various pretreatment methods to overcome recalcitrance are used, including physical methods, chemical methods, biological treatment methods and so on[6-9]. So, the samples used in the experiment are also pretreated.

In addition, cellulose enzyme and glucoside enzyme are used for enzyme hydrolysis. The process of enzyme hydrolysis is maintained at $50^{\circ} \mathrm{C}$ using a shaking incubator. The model for glucose analysis is BIOCHEMISTRY ANALYZER YSI 2700 SELECT.

\section{The Experimental Results and Analysis}

\subsection{The enzymatic hydrolysis of the straws}

The samples of straw cellulose consist of solid content $21.81 \%$ (1\#), $18.23 \%$ (2\#) and $16.48 \%$ (3\#). Three samples are received different pretreatment ways, i.e., sample $1 \#$ is pretreated with concentration $0.4 \%$ (V/V) sulphuric acid, sample $2 \#$ is pretreated with mass concentration $3 \%$ bisulfite, and sample 3\# with concentration $3 \%+0.2 \%(\mathrm{~V} / \mathrm{V})$ bisulfite added sulfuric acid. The enzyme concentration is 7.5 FPU (FPU referring to used enzyme amount to produce $1 \mu \mathrm{mol}$ of glucose in 1 minute), and hydrolysate concentration of samples is $15 \%$. The experimental results are shown in Figure 1.

As showed in Figure 1, due to different pretreatment methods, although the solid content of sample $2 \#$ is greater than the one of sample 3\#, but the sugar yield of sample $2 \#$ is lower than the one of sample 3\#. In addition, experimental results also indicate that, as a whole, the straw contains less sugar. Hence, it brings certain difficulty to produce ethanol fuel massively by the straw, for if we want to gain a certain amount of sugar yield, a mass of straw is needed, and enormous volume of straw will bring difficulty to warehousing and transportation. 


\subsection{The effect of hydrolysis with different hydrolysate concentration and wood cellulose}

Spruce and aspen are chosen as the comparison group. Solid content of sample spruce and aspen cellulose is $34.08 \%$ and $36.3 \%$ respectively and the samples are pretreated by steam-exploded. Hydrolysate concentration of the samples is $2 \% \sim 15 \%$ and the enzyme concentration is 15 FPU. The results are presented in Figure 2.

Figure 2 shows that the sugar yield of aspen is higher than the one of spruce for their distinctive internal structure of lignocelluloses. In addition, higher, the hydrolysate concentration, the higher glucose yield is.

\subsection{The effects of enzyme and hydrolysate concentration on the hydrolysis}

Spruce is used as experimental sample. Its solid content is $34.08 \%$. Hydrolysate concentration is $2 \%$ and $15 \%$, and enzyme concentration is 7.5 FPU and 15 FPU respectively. The experimental results are shown in figure 3 . The results indicate that the enzyme concentration has a certain influence on the sugar yield, but hydrolysate concentration of the sample determines the sugar yield.

\subsection{The comparison of hydrolytic process with high hydrolysate concentration}

Spruce and aspen are still chosen as the comparison group. The solid content of spruce and aspen cellulose is $34.08 \%$ and $36.3 \%$ respectively. Enzyme concentration is $15 \mathrm{FPU}$ and hydrolysate concentration is $20 \%$. The experimental results are shown in figure 4 . As mentioned before the sugar yield of aspen is greater than the one of spruce. The results indicate higher the hydrolysate concentration the slower hydrolytic rate is. The hydrolysis begins after nearly five hours under the concentration. Author performed experiments to hydrolysate concentration of $35 \%$ and found hydrolysis hardly happens for spruce but still occurs for aspen in quite a long time. So how to generate hydrolysis with high hydrolysate concentration of wood cellulose is still needed to be taken into consideration for only with high hydrolysate concentration we can obtain high sugar yield. 


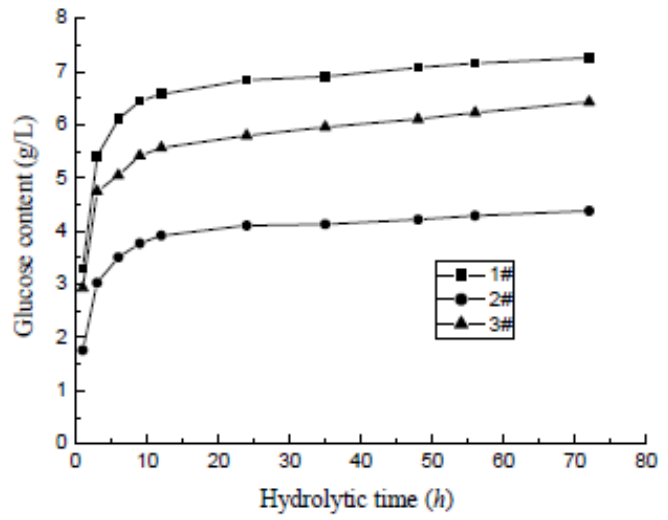

Fig. 1. The hydrolytic process of straw Vs hydrolytic time.

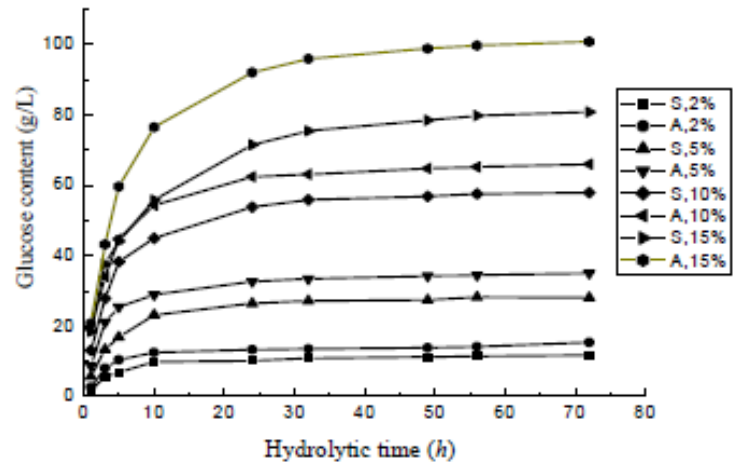

Fig. 2. The hydrolysis of spruce and aspen with different hydrolysate concentration.

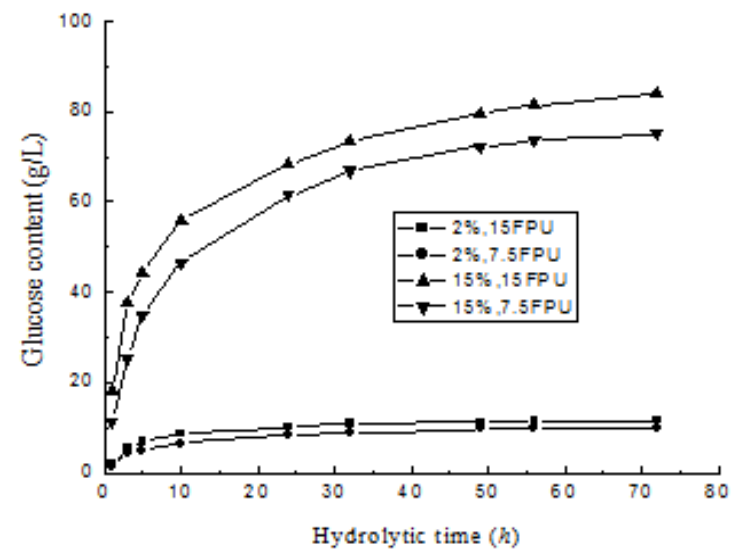

Fig. 3. The hydrolysis of spruce under different FPU and hydrolysate concentration. 


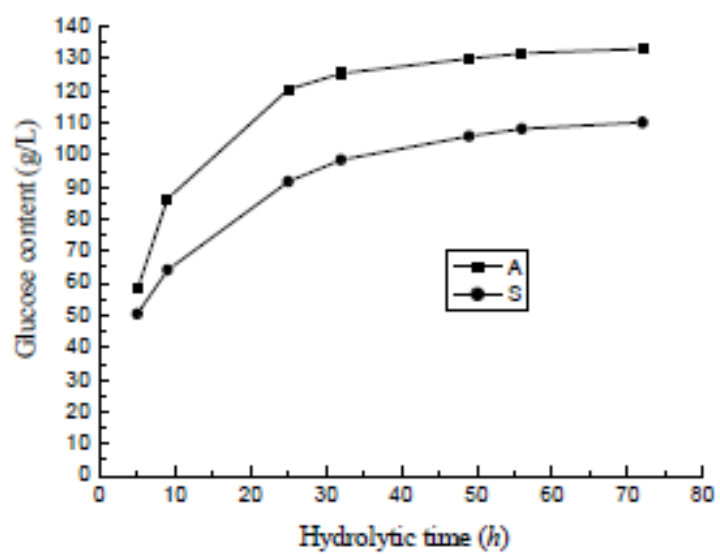

Fig. 4. The hydrolysis of spruce and aspen with high hydrolysate concentration.

\section{Conclusions}

In this paper the effect of hydrolysate concentrations used enzyme concentration and different pretreatment methods on the hydrolysis of different wood cellulose is studied experimentally. The results of the hydrolysis of straw show that different pretreatment methods have great influence on the hydrolytic efficiency, so finding and optimizing pretreatment methods to improve the hydrolytic efficiency is of critical significance. In addition the sugar yield of various lignocelluloses is also different for its distinctive inner structure. Furthermore, the use of enzymes has an important impact on accelerating hydrolytic process and increasing glucose yield. Above all finding and optimizing hydrolytic ways especially for the hydrolysis of high hydrolysate concentration and reducing costs in the hydrolytic process is still of great significance.

\section{References}

1. E.D. Bruce Lignocellulose conversion and the future of fermentation biotechnology [J]. TIBTECH-OCTOBER 1987 5(2):287-291.

2. L.Q. Yu Studies on the Factors Affecting Cellulose Hydrolysis and on the Technique of Producing Ethanol [J]. WORLD FORESTRY RESEARCH 2000 13(2) 30-35.

3. D.C. GONG Y.H.TIAN D.Y. LI et al. Advances of Research on Bioethanol from Ligncellnlos [J]. CHEMISTRY \& BIOENGINEERING 2007 24(1): 4-6.

4. W.J. Frederick S. J. Lien C.E. Courchene et al. Production of ethanol from carbohydrates from loblolly pine: a technical and economic assessment [J]. Bioresour Technol 2008 99(11):5051-5057. 
5. D. WANG C.CHANG Progress in enzymatic hydrolysis of cellulosic biomass and the fermentation production of ethanol [J]. CHINESE JOURNAL OF BIOPROCESS ENGINEERING 2010 8(4) 72-77.

6. N. Mosier C. Wyman B. Dale et al. Feature of promising technologies for pretreatment of lignocellulosic biomass [J]. Bioresource Technology 2005 96(6): 673-686.

7. E. H. Michael S.Y. Ding K. J. David et al. Biomass recalcitrance: engineering plants and enzymes for biofuels production [J]. Science 2007 315(5813): 804-807.

8. H.G. Ji H.Pang R.L.Zhang et al. Pretreatment and Enzymatic Hydrolysis of Lignocellulose [J]. CHEMISTRY 2008 71(5): 329-335.

9. H.Fan R. Art Pretreatment and Lignocellulosic Chemistry [J]. BioEnergy Research 2012 5(4):1043-1066. 